\title{
A Multicenter Study of Three-dimensional Echocardiographic Evaluation of Normal Pediatric Left Ventricular Volumes and Function with Automated Versus Semi-Automated Quantification
}

Pei-Ni Jone ${ }^{1}$, Lisa Le ${ }^{1}$, Zhaoxing Pan $^{2}$, Tim Colen ${ }^{3}$, Sachie Shigemitsu ${ }^{3}$, Nee Scze Khoo ${ }^{3}$, Benjamin Goot ${ }^{4}$, Anitha Parthiban ${ }^{5}$, David Harrild ${ }^{6}$, Alessandra Ferraro ${ }^{7}$, and Gerald $\operatorname{Marx}^{6}$

${ }^{1}$ Children's Hospital Colorado

${ }^{2}$ University of Colorado Denver Children's Hospital Colorado Research Institute

${ }^{3}$ Stollery Children's Hospital

${ }^{4}$ Childrens Wisconsin

${ }^{5}$ Children's Mercy Hospitals and Clinics

${ }^{6}$ Boston Children's Hospital

${ }^{7}$ Boston Children s Hospital

October 13, 2020

\begin{abstract}
Background: Three-dimensional echocardiography (3DE) evaluation of left ventricular (LV) volume and function in pediatrics compares favorably with cardiac magnetic resonance imaging. A multicenter trial with automated and semi-automated LV quantification allows for generation of normative data in large pediatric patients. The aims of this study were to evaluate the feasibility and reproducibility of measuring three-dimensional echocardiography (3DE) volumes and function in pediatric patients in a multicenter trial; to determine if automated software (without contouring edits) will improve the reproducibility in volume and function analysis; and thus establish normal z score values in this unique population. Methods: Six hundred and ninety-eight healthy children (ages 0 to 18 years) were recruited from 5 centers. Left ventricular (LV) 3DE was acquired from the 4-chamber view. A vendor independent software analyzed end-diastolic volume (EDV), end-systolic volume (ESV), stroke volume (SV), and ejection fraction (EF) using automated and semi-automated quantification. Feasibility and reproducibility were assessed. Body surface area (BSA) based z-scores were generated. Results: Feasibility was 79\% (523/658). Reproducibility was good between centers using the semi-automated quantification. Reproducibility was decreased using the automated quantification. Therefore, Z-scores were generated for ESV, EDV, and SV using the semi-automated method. Conclusions: $3 \mathrm{DE}$ can reliably evaluate LV volumes and EF in pediatric patients at different centers. We report pediatric Z-scores for normal LV volumes using the semi-automated method. Further optimization of technology will be necessary for reliable use of fully automated quantification by $3 \mathrm{DE}$ in children.
\end{abstract}

A Multicenter Study of Three-dimensional Echocardiographic Evaluation of Normal Pediatric Left Ventricular Volumes and Function with Automated Versus Semi-Automated Quantification

Pei-Ni Jone MD ${ }^{1}$, Lisa Le RDCS ${ }^{1}$, Zhaoxing Pan $\mathrm{PhD}^{2}$, Tim Colen $\mathrm{MBBS}^{3}$, Sachie Shigemitsu $\mathrm{MD}^{3}$, Nee Khoo MBBS ${ }^{3}$, Benjamin H. Goot MD4, Anitha Parthiban MD5, David M. Harrild MD, PhD6, Alessandra M. Ferraro MD6, Gerald Marx MD6 
${ }^{1}$ Pediatric Cardiology, Children's Hospital Colorado, University of Colorado School of Medicine, Aurora, Colorado

${ }^{2}$ Research Institute, Children's Hospital Colorado, University of Colorado School of Medicine, Aurora, Colorado

${ }^{3}$ Stollery Children's Hospital, University of Alberta, Edmonton, Canada

4Herma Heart Institute, Children's Wisconsin, Medical College of Wisconsin, Milwaukee, Wisconsin

5Children's Mercy Hospital, University of Missouri, Kansas City, Missouri

6Boston Children's Hospital, Harvard Medical School, Boston, Massachusetts

Disclosures: The authors report no disclosures.

\section{Corresponding Author:}

Pei-Ni Jone MD

Mailing Address:

13123 East $16^{\text {th }}$ Avenue, B100, Aurora, Colorado 80045

Telephone: 720-777-2944

E-mail: pei-ni.jone@childrenscolorado.org

Background: Three-dimensional echocardiography (3DE) evaluation of left ventricular (LV) volume and function in pediatrics compares favorably with cardiac magnetic resonance imaging. A multicenter trial with automated and semi-automated LV quantification allows for generation of normative data in large pediatric patients. The aims of this study were to evaluate the feasibility and reproducibility of measuring threedimensional echocardiography (3DE) volumes and function in pediatric patients in a multicenter trial; to determine if automated software (without contouring edits) will improve the reproducibility in volume and function analysis; and thus establish normal z score values in this unique population.

Methods: Six hundred and ninety-eight healthy children (ages 0 to 18 years) were recruited from 5 centers. Left ventricular (LV) 3DE was acquired from the 4-chamber view. A vendor independent software analyzed end-diastolic volume (EDV), end-systolic volume (ESV), stroke volume (SV), and ejection fraction (EF) using automated and semi-automated quantification. Feasibility and reproducibility were assessed. Body surface area (BSA) based z-scores were generated.

Results: Feasibility was 79\% (523/658). Reproducibility was good between centers using the semiautomated quantification. Reproducibility was decreased using the automated quantification. Therefore, Z-scores were generated for ESV, EDV, and SV using the semi-automated method.

Conclusions: 3DE can reliably evaluate LV volumes and EF in pediatric patients at different centers. We report pediatric Z-scores for normal LV volumes using the semi-automated method. Further optimization of technology will be necessary for reliable use of fully automated quantification by 3DE in children.

Key words: Three dimensional echocardiography, left ventricular volume, left ventricular function, normal data

\section{INTRODUCTION}

Quantification of left ventricular size and function from echocardiography is important in the diagnosis, prognosis, and management of pediatric heart disease. ${ }^{1}$ With rapid improvement in technology, three-dimensional echocardiography (3DE) evaluation of left ventricular (LV) volume and function in pediatrics compares favorably with magnetic resonance imaging $(\mathrm{MRI})^{2-5}$. However, a multicenter trial, to assess reproducibility, has not been done in the pediatric population. 
Recent technological advances allow fully automated quantification (without edits to LV contouring borders) of left heart chamber by $3 \mathrm{DE}$, thus potentially reducing the time and difficulty, and potentially increasing the reliability of the LV volume quantification. Automated contouring might enhance the adoption of 3DE quantification of LV that can be incorporated into routine clinical practice.

A multicenter trial would provide important data concerning the reproducibility of 3DE to measure LV volumes and function. Such a large-scale study would also allow for generation of normative data in pediatric patients. Moreover, such a study could also evaluate the differences between automated and semi-automated quantification software. Thus, the aims of this study were to evaluate the feasibility and reproducibility of measuring 3D volumes and function in young pediatric patients in a multicenter trial; and to determine if automated software (without contouring edits) will improve the reproducibility in volume and function analysis; and thus establish normal z score values in this unique population.

\section{METHODS:}

\section{Study population}

Three-dimensional LV datasets were obtained from healthy subjects from 5 pediatric centers from 20142019. Inclusion criteria were normal subjects (ages 0 to 18 years) who presented for routine clinical care for heart murmur, chest pain, or syncope. These subjects had echocardiographic evidence of structurally and functionally normal hearts. Exclusion criteria were similar to the Pediatric Heart Network normal echocardiographic database. ${ }^{6}$ Patients with cardiac abnormalities (except for patent foramen ovale, trivial branch pulmonary stenosis, or inaudible patent ductus arteriosus), Kawasaki disease, cardiomyopathy, exposure to anthracyclines, cardiac transplantation, chronic systemic disease, and preterm infants $<37$ weeks gestation were excluded. Consents and waiver of consents were obtained based on the individual institutional ethics boards and data use agreements were obtained from each center to Children's Hospital Colorado (Center 1) which served as the core laboratory for the study. All 3D datasets were anonymized and sent to the core laboratory for data analysis.

\section{$3 D$ dataset acquisition and analysis}

Real-time 3DE image acquisitions of the LV from the 4-chamber view were performed using echocardiographic ultrasound platforms (GE Vivid E9/E95; Vingmed Ultrasound, Horten, Norway, iE33/EPIQ; Philips Medical Systems, Andover, MA, USA, and/or Siemens SC2000, Siemens Medical Solutions, Malvern, PA, USA). 3D datasets were excluded by the core lab if any portion of $\mathrm{LV}$ endocardial borders or $\mathrm{LV}$ apex were missing from the 3D dataset. Studies were also excluded if the vendor independent software (4D LV Analysis, TomTec Imaging Systems version 4.0, Unterschleissheim, Germany) was not able to automatically track the borders of the LV after three attempts. At each center (with the exception of center 5), the fully automated method was first used to generate LV end diastolic and end systolic volumes (EDV and ESV), stroke volumes (SV), and ejection fraction (EF) without editing the borders of the LV. Timing from the start to the end of the analysis was recorded. Then semi-automated analysis was performed by all centers on the same datasets, manually editing the contours to define the LV endocardial borders for the same variables. Timing from the start to the end of the analysis was again recorded. All LV EDV, ESV, SV, and EF data points from each center were entered into REDCap located at the core laboratory. Subsequently, all 3DE datasets were transferred to the core laboratory and had the LV volumes and EF re-measured using the fully automated and semi-automated methods described above. The core laboratory observer was blinded to the reported measures in REDCap to determine intercenter reproducibility. The core laboratory then repeated analysis for intraobserver and interobserver variability 6 months after initial analysis in 86 randomly selected studies at the core lab.

\section{Statistical analysis}

Continuous data were expressed as mean \pm standard deviation (SD) or median and interquartile range (IQR). Reproducibility within core lab and between centers was compared by Bland-Altman plot and percent difference was calculated for LV EDV, ESV, and SV as difference of the two measures divided by the 
mean of the two measures. Time required to complete fully automated and semi-automated contouring were compared between centers using analysis of variance (ANOVA) and as a group using student t-test. BSA was calculated using Haycock's method ${ }^{7}$. To build the z-model of a parameter (i.e. ESV, DSV and SV), we selected an optimum exponent, $\alpha$, of the index parameter (parameter/BSA ${ }^{\alpha}$ ) such that: 1.) The index parameter satisfactorily follows a normal distribution and 2.) The index parameter does not depend upon BSA. Z-score was then calculated as

$Z=\frac{\left[\left(\frac{\text { parameter }}{\mathrm{BSA}^{\alpha}}\right)-(\text { mean value of indexed parameter })\right]}{\text { SD of indexed parameter }}{ }^{6}$ Normality of an indexed parameter was evaluated using Shapiro-Wilk and Kolmogorov-Smirnov tests, Q-Q plot, skewness and kurtosis. Dependence of the indexed parameter on BSA was evaluated with a test of the slope of the linear regression of the indexed parameters on BSA. We conducted grid search with a 0.001 step size to find the optimum exponent, $\alpha$, and chose the one that maximized the sum of p-value for Shapiro-Wilk test and the p-value of testing the slope of index parameter vs. BSA. During the model development, diagnostic analysis were conducted using leave-one-out method. Few data points with extreme values that influences the distribution of indexed parameter were excluded from the final z-model development. After the optimum has determined, association of indexed parameter with age and gender were further examined with respectively linear regression and Student t-test. Gender specific z-scores model hence developed because there is difference between genders in indexed parameter. A two-sided p-value $<0.05$ was considered statistically significant. Statistical analysis was performed using SAS version 9.4 (SAS institute, Cary, NC).

\section{RESULTS:}

\section{Study Subjects}

Six hundred fifty-eight subject studies were sent to the core lab for analysis. Five hundred twenty-three (79\%) LV 3D datasets could reliably be traced at the core lab using the automated and semi-automated method. The median (IQR) patient age was 10 (5 to 14) years. Eighteen percent (96/523) of the patients were [?] 3 years of age. The mean BSA was $1.2 \pm 0.5 \mathrm{~m}^{2}$. There were 291 males $(56 \%)$ and 232 females $(44 \%)$. The age range and feasibility of study subjects in different age groups are reported in Table 1.

\section{Comparison of automated versus semi-automated methods}

Five hundred and thirty-eight datasets were used for automated and semi-automated methods comparison from all centers. Bland-Altman analysis showed wide limit of agreement (LOA) between the automated versus semi-automated contouring of the 3D LV datasets. The bias (LOA) for EDV, ESV, SV, and EF were $-0.56 \%$ (-32.25 to 31.13), 1.01 (-29.94 to 31.96), -1.73 (-18.12 to 14.65), and -1.52 (-17.05 to 14.01) respectively (Table 2). Sub-analysis of the inter-center reproducibility for LV volumes and function between the core lab and other centers using the automated method showed a bigger bias and LOA than the semi-automated methods (Table 2). The inter-center reproducibility for LV volumes and EF showed less bias and LOA between the core lab and other centers using semi-automated contouring (Figure 1 and Table 2). The percent difference using the automated and semi-automated methods for LV EDV, ESV, and SV were summarized in Figure 2. The mean percentage difference in EDV and ESV are higher using the automated method compared to the semi-automated method. The mean absolute percent difference in 3D LV EF between all centers was $7.4 \pm 5.4 \%$. Figure 3 shows the Bland-Altman analysis percent difference of the core lab and other centers using the semi-automated method in ages 0-4 years, 5-9 years, 10-14 years, and 15-18 years of age respectively.

Automated versus semi-automated contour timing

Automated contouring of the LV 3D datasets was faster than semi-automated contouring. The mean $\pm \mathrm{SD}$ (minutes) in automated contouring in in Centers 1-4 were $0.19 \pm 0.02,0.56 \pm 0.16,0.19 \pm 0.06$, and $0.32 \pm$ 0.09 minutes respectively $(\mathrm{p}<0.001)$. The mean $\pm \mathrm{SD}$ (minutes) in semi-automated contour timing in Center $1-5$ were $2.66 \pm 0.63,2.39 \pm 0.78,3.35 \pm 1.04,3.48 \pm 1.03$, and $2.82 \pm 0.66$ minutes respectively $(\mathrm{p}<0.001)$.

Core Lab Intraobserver Reproducibility of Automated and Semi-automated Methods

The intraobserver variability of 86 studies using automated and semi-automated methods in the core lab 
was excellent and shown in Table 3. The bias was small in all variables using each of the individual methods. The LOA of the automated versus semiautomated contouring of the LV size and function were very wide.

\section{Z-score generation}

Normative pediatric z-score generation was performed on the 523 LV 3D datasets using the semi-automated method. The optimum exponents are shown in Table 4 as well as the mean and SD of indexed parameters. Figure 4 demonstrates the normative data and z-score for ESV, EDV, and SV derived from the semiautomated method.

\section{DISCUSSION}

This study reports the largest multicenter data collection for development of 3D echocardiographic z-scores for LV volumes and function in healthy North American children using the semi-automated quantification method. The feasibility was $79 \%$ analyzable datasets at the core laboratory. We demonstrated that the semi-automated software quantification with LV border adjustment was more reliable between centers than automated software quantification. Although automated software quantification took less time, the greater variability in measurement rendered it less reliable, and therefore the normative Z-score values were derived from the semi-automated method.

Our study had feasibility of $79 \%$ of obtaining adequate 3D data for analysis at the core laboratory, similar to another multicenter study in the pediatric age group ${ }^{8}$. Krell et al reported having a feasibility of $74 \%$ in their multicenter study but our study population was larger with more patients. Kuebler et al reported normative LV volume and functional values in 238 pediatric subjects of different age group and body surface area ${ }^{9}$. Only $14 \%$ of their subjects were under the age of $5(34 / 238)^{9}$. Our study is notable in that $27 \%$ $(141 / 523)$ of the subjects were under the age of 5 and $18 \%$ were less than 3 years old. This is the population that is technically challenging to acquire $3 \mathrm{D}$ volumes as young patients are unable to hold their breath or stay relatively motionless during the acquisition, obviating "stitch artifact". On the other hand, they may have the best imaging window due to smaller chest walls and ability to incorporate the entire ventricular volume into the dataset. Cantinotti et al studied 800 Italian healthy children and reported excellent feasibility of 91\%; however, the feasibility for smaller children with smaller BSA less than 0.5 was $68 \%$ to $80 \%$ respectively ${ }^{10}$.

We did not find that the automated quantification software improved the reproducibility in volume and function analysis. There was wide LOA between automated and semiautomated LV quantification in all centers. The automated software frequently did not track the LV endocardial borders. This became apparent in the intraobserver reproducibility in the core lab when the same LV datasets were re-measured using the semi-automated contouring method. The intraobserver reproducibility was excellent when semi-automated method was used and showed small bias and LOA. However, the intraobserver reproducibility did not have good agreement when automated versus semi-automated quantification comparison was used on the same LV datasets. This study highlights the difference between pediatric and adult studies of using automated versus semi-automated contouring of LV volumes and function. We postulate that the current automated contouring does not adequately track LV contours in pediatric 3D datasets because the fully automated algorithms are built based on adult 3D datasets. The time for semi-automated contouring of the LV was not more than a mean of 2-3 minutes in all centers, which should be acceptable for routine clinical use.

Our study is unique in that we performed extensive analysis of reproducibility by comparing all 3D datasets from 4 different centers to the core lab using the semi-automated and automated methods. The semi-automated methods between centers were reproducible. The percent difference in the LV EDV was \pm $30 \%$ indicating that if an individual measured the LV EDV at $10 \mathrm{ml}$, the difference could be $\pm 3 \mathrm{ml}$. This is an acceptable range of measures between centers. The percent difference for LV ESV measurement was also about $\pm 30 \%$ (Figure 3). The LOA of LV volumes and EF were wider using the automated methods compared to the semi-automated methods between the core lab to other centers, indicating that LV volumes and EF were not reproducible using this method. Prior studies from Kuebler et al and Cantinotti et al have described pediatric normative LV volumes and function derived from single centers ${ }^{9,10}$. Similar to previous studies, LV volumes indexed to the BSA demonstrated gradual increase from childhood to adolescent years 
$8-12$.

The mean absolute percent difference in 3D LV EF between all centers in our study was $7 \%$ with the observed difference of $4.2 \%$ if the LV EF was measured at $60 \%$. This is better than the reported $2 \mathrm{D}$ LV EF absolute percentage difference of $11.2 \%$ in the Pediatric Heart Network study ${ }^{13}$. 3D LV EF has been reported to be more accurate and reproducible than $2 \mathrm{D} \mathrm{LV} \mathrm{EF} \mathrm{in} \mathrm{adults} \mathrm{and} \mathrm{children} \mathrm{because} \mathrm{3DE} \mathrm{does} \mathrm{not} \mathrm{rely} \mathrm{on}$ geometric assumptions and is less affected by acquisition technique such as foreshortening ${ }^{3,14-18}$. Thus, it is recommended that $3 \mathrm{D} \mathrm{LV} \mathrm{EF} \mathrm{should} \mathrm{be} \mathrm{reported} \mathrm{in} \mathrm{clinical} \mathrm{echocardiograms} \mathrm{in} \mathrm{centers} \mathrm{with} \mathrm{experience} \mathrm{in}$ $3 \mathrm{DE}{ }^{18}$. Our study demonstrated that the reproducibility of $3 \mathrm{D} \mathrm{LV} \mathrm{EF} \mathrm{is} \mathrm{good} \mathrm{to} \mathrm{excellent} \mathrm{and} \mathrm{can} \mathrm{be} \mathrm{used}$ in multiple centers.

\section{Limitations}

The limitation of the study includes race and ethnicity were not reported because of the limited data use agreement among centers in which this information could not be collected. The 1 to 2 year-old patients were difficult to recruit as they were the most uncooperative patients and frequently cannot breath hold for image acquisition. We did not record the amount of time we spent in acquiring the LV 3D datasets as this can be quite variable depending on the state of the child's cooperation. However, the post-processing using semi-automated contouring is feasible once a good dataset is acquired and does not consume large amount of time making this clinically feasible to use.

\section{CONCLUSION}

Generation of normal pediatric z-scores for LV volumes and function from 3DE is feasible, and these values can serve as a reference for pediatric patients. Semi-automated contouring for the LV in pediatrics is reliable and reproducible. Agreement between centers was good and supports between-center comparison of LV volumes and EF. Further optimization of technology is necessary for reliable use of fully automated quantification by $3 \mathrm{DE}$ in children.

\section{Sources of Funding: None REFERENCES}

1. Lopez L, Colan SD, Frommelt PC, et al. Recommendations for quantification methods during the performance of a pediatric echocardiogram: a report from the Pediatric Measurements Writing Group of the American Society of Echocardiography Pediatric and Congenital Heart Disease Council. J Am Soc Echocardiogr. 2010;23(5):465-495; quiz 576-467.

2. Shimada YJ, Shiota T. A meta-analysis and investigation for the source of bias of left ventricular volumes and function by three-dimensional echocardiography in comparison with magnetic resonance imaging. $A m \mathrm{~J}$ Cardiol. 2011;107(1):126-138.

3. Dorosz JL, Lezotte DC, Weitzenkamp DA, Allen LA, Salcedo EE. Performance of 3-dimensional echocardiography in measuring left ventricular volumes and ejection fraction: a systematic review and meta-analysis. J Am Coll Cardiol. 2012;59(20):1799-1808.

4. Friedberg MK, Su X, Tworetzky W, Soriano BD, Powell AJ, Marx GR. Validation of 3D echocardiographic assessment of left ventricular volumes, mass, and ejection fraction in neonates and infants with congenital heart disease: a comparison study with cardiac MRI.Circ Cardiovasc Imaging. 2010;3(6):735-742.

5. Simpson J, Lopez L, Acar P, et al. Three-dimensional echocardiography in congenital heart disease: an expert consensus document from the European Association of Cardiovascular Imaging and the American Society of Echocardiography. Eur Heart J Cardiovasc Imaging.2016;17(10):1071-1097.

6. Lopez L, Colan S, Stylianou M, et al. Relationship of Echocardiographic Z Scores Adjusted for Body Surface Area to Age, Sex, Race, and Ethnicity: The Pediatric Heart Network Normal Echocardiogram Database. Circ Cardiovasc Imaging. 2017;10(11). 
7. Haycock GB, Schwartz GJ, Wisotsky DH. Geometric method for measuring body surface area: a heightweight formula validated in infants, children, and adults. J Pediatr. 1978;93(1):62-66.

8. Krell K, Laser KT, Dalla-Pozza R, et al. Real-Time Three-Dimensional Echocardiography of the Left Ventricle-Pediatric Percentiles and Head-to-Head Comparison of Different Contour-Finding Algorithms: A Multicenter Study. J Am Soc Echocardiogr. 2018;31(6):702-711 e713.

9. Kuebler JD, Ghelani S, Williams DM, et al. Normal Values and Growth-Related Changes of Left Ventricular Volumes, Stress, and Strain in Healthy Children Measured by 3-Dimensional Echocardiography. Am $J$ Cardiol. 2018;122(2):331-339.

10. Cantinotti M, Scalese M, Giordano R, et al. Three-Dimensional Echocardiography Derived Nomograms for Left Ventricular Volumes in Healthy Caucasian Italian Children. J Am Soc Echocardiogr.2019;32(6):794797 e791.

11. Buccheri S, Costanzo L, Tamburino C, Monte I. Reference Values for Real Time Three-Dimensional Echocardiography-Derived Left Ventricular Volumes and Ejection Fraction: Review and Meta-Analysis of Currently Available Studies. Echocardiography. 2015;32(12):1841-1850.

12. Hascoet S, Brierre G, Caudron G, Cardin C, Bongard V, Acar P. Assessment of left ventricular volumes and function by real time three-dimensional echocardiography in a pediatric population: a TomTec versus QLAB comparison. Echocardiography. 2010;27(10):1263-1273.

13. Frommelt PC, Minich LL, Trachtenberg FL, et al. Challenges With Left Ventricular Functional Parameters: The Pediatric Heart Network Normal Echocardiogram Database. J Am Soc Echocardiogr.2019;32(10):1331-1338 e1331.

14. Muraru D, Badano LP, Peluso D, et al. Comprehensive analysis of left ventricular geometry and function by three-dimensional echocardiography in healthy adults. J Am Soc Echocardiogr. 2013;26(6):618-628.

15. Fukuda S, Watanabe H, Daimon M, et al. Normal values of real-time 3-dimensional echocardiographic parameters in a healthy Japanese population: the JAMP-3D Study. Circ J. 2012;76(5):1177-1181.

16. Bernard A, Addetia K, Dulgheru R, et al. 3D echocardiographic reference ranges for normal left ventricular volumes and strain: results from the EACVI NORRE study. Eur Heart J Cardiovasc Imaging.2017;18(4):475-483.

17. Kaku K, Takeuchi M, Otani K, et al. Age- and gender-dependency of left ventricular geometry assessed with real-time three-dimensional transthoracic echocardiography. J Am Soc Echocardiogr.2011;24(5):541-547.

18. Lang RM, Badano LP, Mor-Avi V, et al. Recommendations for cardiac chamber quantification by echocardiography in adults: an update from the American Society of Echocardiography and the European Association of Cardiovascular Imaging. Eur Heart J Cardiovasc Imaging.2015;16(3):233-270.

Tables

Table 1: Feasibility of study subjects in different age group

\begin{tabular}{llll}
\hline Age (years) & Traceable datasets $(\mathrm{N}=523) \mathrm{M} / \mathrm{F}(291 / 232)$ & Total in age group $(\mathrm{N}=658)$ & Feasibility in age group (\%) \\
\hline$<1$ & 51 & 66 & 77.3 \\
1 & 6 & 9 & 66.7 \\
2 & 14 & 19 & 73.7 \\
3 & 25 & 35 & 71.4 \\
4 & 15 & 17 & 88.2 \\
5 & 30 & 38 & 78.9 \\
6 & 24 & 28 & 85.7 \\
7 & 21 & 27 & 77.8
\end{tabular}




\begin{tabular}{llll}
\hline Age $($ years $)$ & Traceable datasets $(\mathrm{N}=523) \mathrm{M} / \mathrm{F}(291 / 232)$ & Total in age group $(\mathrm{N}=658)$ & Feasibility in age group (\%) \\
\hline 8 & 18 & 21 & 85.7 \\
9 & 35 & 45 & 77.8 \\
10 & 28 & 34 & 82.4 \\
11 & 34 & 39 & 87.2 \\
12 & 31 & 41 & 75.6 \\
13 & 37 & 42 & 88.1 \\
14 & 37 & 49 & 75.5 \\
15 & 41 & 49 & 83.7 \\
16 & 30 & 39 & 76.9 \\
17 & 30 & 40 & 75.0 \\
18 & 16 & 20 & 80.0 \\
\hline
\end{tabular}

$\mathrm{M}=$ male, $\mathrm{F}=$ female

Table 2: Bland-Altman analysis of automated and semi-automated measurements of left ventricular volumes and function in all centers, and Bland-Altman analysis of automated and semi-automated methods between core lab and other centers.

\begin{tabular}{llll}
\hline & $\begin{array}{l}\text { Auto vs Semi All } \\
\text { centers }\end{array}$ & $\begin{array}{l}\text { Auto Center 1 vs all } \\
\text { centers }\end{array}$ & $\begin{array}{l}\text { Semi Center 1 vs all } \\
\text { centers }\end{array}$ \\
\hline EDV (ml) & & & \\
Bias 95\% LOA p & $-0.56-32.25$ to 31.13 & $\begin{array}{l}-3.85-38.93 \text { to } 31.22 \\
<0.001\end{array}$ & $0.46-23.86$ to 24.77 \\
& 0.423 & $-4.21-42.01$ to 33.59 & $2.56-10.81$ to 15.94 \\
ESV (ml) & $1.01-29.94$ to 31.96 & $<0.001$ & $<0.001$ \\
Bias 95\% LOA p & 0.140 & $-0.36-12.20$ to 12.92 & $-2.19-19.55$ to 15.17 \\
& & 0.299 & $<0.001$ \\
SV (ml) & $-1.73-18.12$ to 14.65 & $4.16-9.86$ to 18.17 & $-3.11-12.67$ to 6.44 \\
Bias 95\% LOA p & $<0.001$ & $<0.001$ & $<0.001$ \\
\hline EF (\%) & $-1.52-17.05$ to 14.01 & & \\
Bias 95\% LOA p & $<0.001$ &
\end{tabular}

$\mathrm{EDV}=$ end-diastolic volume, $\mathrm{EF}=$ ejection fraction, $\mathrm{ESV}=$ end-systolic volume, $\mathrm{LOA}=$ limit of agreement, $\mathrm{SD}=$ standard deviation, $\mathrm{SV}=$ stroke volume.

Table 3: Intraobserver variability of semi-automated and automated measurements of left ventricular volumes and function at core lab using Bland-Altman analysis.

\begin{tabular}{llll}
\hline Center 1 (core lab) & $\begin{array}{l}\text { Semi 1 versus Semi } 2 \\
(\mathrm{n}=86)\end{array}$ & $\begin{array}{l}\text { Auto 1 versus Auto 2 } \\
(\mathrm{n}=86)\end{array}$ & $\begin{array}{l}\text { Auto 1 versus Semi 1 } \\
(\mathrm{n}=86)\end{array}$ \\
\hline EDV $(\mathbf{m l})$ & & & \\
Bias 95\% LOA p & $-1.77-7.73$ to 2.12 & $-0.03-0.64$ to 0.58 & $2.22-19.05$ to 23.49 \\
& $<0.001$ & 0.360 & 0.061 \\
ESV $(\mathbf{m l})$ & & $-0.26-4.92$ to 4.40 & $2.52-12.31$ to 17.35 \\
Bias 95\% LOA p & $-0.75-4.71$ to 3.21 & 0.312 & 0.003 \\
SV $(\mathbf{m l})$ & $<0.001$ & &
\end{tabular}




\begin{tabular}{llll}
\hline Center 1 (core lab) & $\begin{array}{l}\text { Semi 1 versus Semi } 2 \\
(\mathrm{n}=86)\end{array}$ & $\begin{array}{l}\text { Auto 1 versus Auto 2 } \\
(\mathrm{n}=86)\end{array}$ & $\begin{array}{l}\text { Auto 1 versus Semi } 1 \\
(\mathrm{n}=86)\end{array}$ \\
\hline Bias 95\% LOA p & $\begin{array}{l}-1.02-5.76 \text { to } 3.72 \\
<0.001\end{array}$ & $0.23-3.83$ to 4.280 .313 & $-0.30-15.58$ to 14.98 \\
& & & 0.722 \\
EF (\%) & $-0.06-4.37$ to 4.49 & $0.33-5.53$ to 6.180 .313 & $-3.00-21.13$ to 15.13 \\
Bias 95\% LOA p & 0.807 & & 0.003 \\
\hline
\end{tabular}

$\mathrm{EDV}=$ end-diastolic volume, $\mathrm{EF}=$ ejection fraction, $\mathrm{ESV}=$ end-systolic volume, $\mathrm{LOA}=$ limit of agreement, $\mathrm{SD}=$ standard deviation, $\mathrm{SV}=$ stroke volume.

Table 4: Parameters for calculating z-scores.

\begin{tabular}{lllll}
\hline Parameter & Exponent $\alpha$ & Gender & $\begin{array}{l}\text { Mean of index } \\
\text { parameter }\end{array}$ & $\begin{array}{l}\text { SD of index } \\
\text { parameters }\end{array}$ \\
\hline ESV & 1.284 & F and M & 22.744 & 4.422 \\
& & F & 21.491 & 3.936 \\
EDV & \multirow{2}{*}{1.277} & F & 23.768 & 4.538 \\
& & F and M & 56.874 & 10.124 \\
& & M & 53.729 & 8.705 \\
SV & \multirow{2}{*}{1.278} & F and M & 59.416 & 10.485 \\
& & F & 34.073 & 6.230 \\
& & M & 32.237 & 5.497 \\
& & 35.547 & 6.398 \\
\hline
\end{tabular}

Index parameter $=$ Parameter $/ \mathrm{BSA}{ }^{\alpha} . \mathrm{EDV}=$ end-diastolic volume, $\mathrm{ESV}=$ end-systolic volume, $\mathrm{F}=$ female, $\mathrm{M}=$ male, $\mathrm{SV}=$ stroke volume

\section{Figure Legends}

Figure 1: Interobserver variability between the core lab and other centers using the semi-automated method. Bland-Altman analysis plots are shown for LV EDV, ESV, SV, and EF. EDV = end-diastolic volume, EF = ejection fraction, $\mathrm{ESV}=$ end-systolic volume, $\mathrm{LV}=$ left ventricle, $\mathrm{SV}=$ stroke volume.

Figure 2: Percent difference calculated for LV EDV, ESV, and SV between core lab versus other centers using the semi-automated and automated methods. EDV $=$ end-diastolic volume, ESV = end-systolic volume, $\mathrm{LV}=$ left ventricle, $\mathrm{SV}=$ stroke volume.

Figure 3: Bland-Altman analysis (percent difference) of left ventricular parameters in core lab versus other centers in all ages, and break down of 0-4 years of age, 5-9 years of age, 10-14 years of age, and 15-18 years of age.

Figure 4: Normative Z-score values for left ventricular volumes. BSA = body surface area, EDV = enddiastolic volume, $\mathrm{ESV}=$ end-systolic volume, $\mathrm{SV}=$ stroke volume. 
Semi-automated Method: Inter-center Reproducibility Between Core Lab and Other Centers
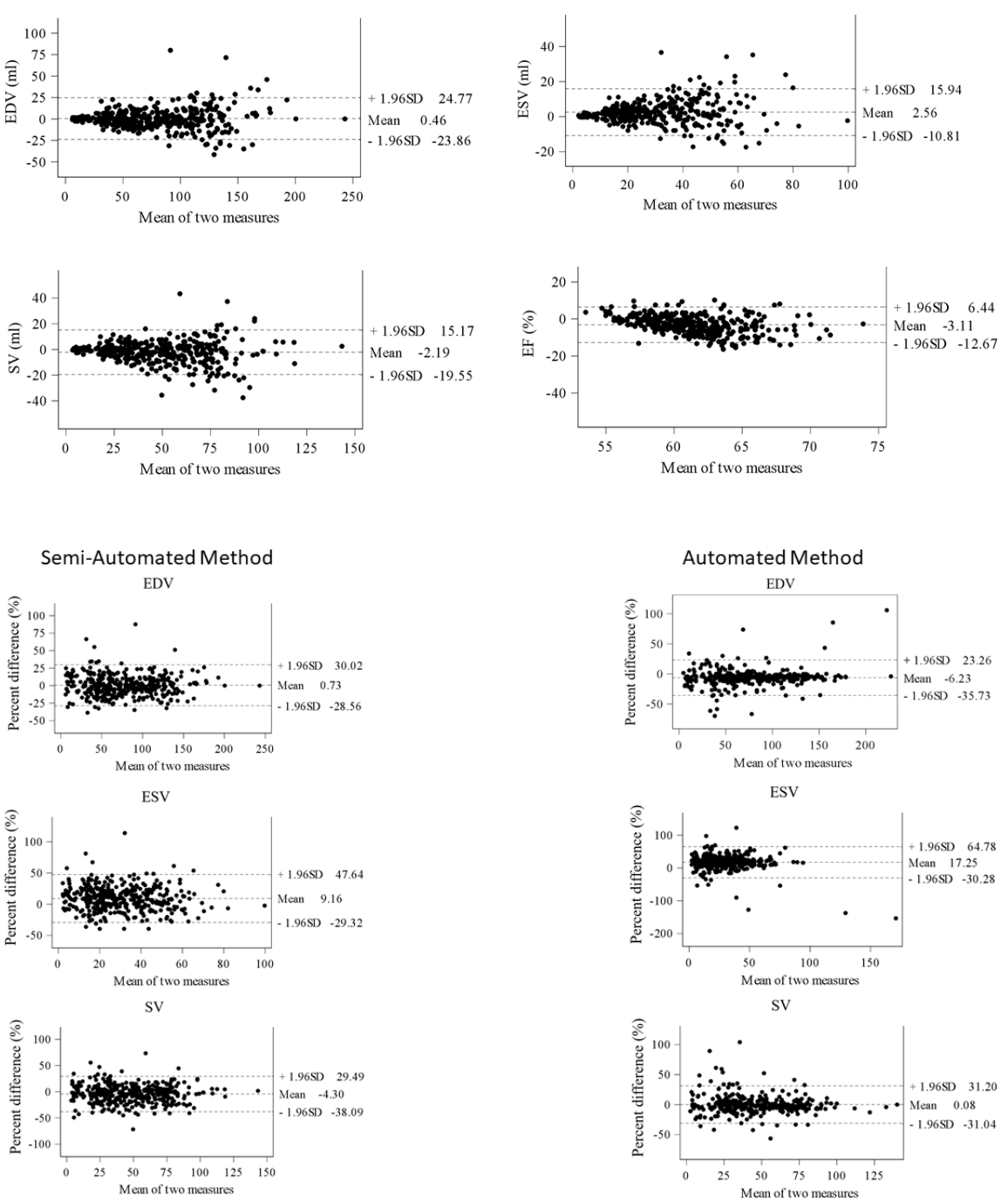

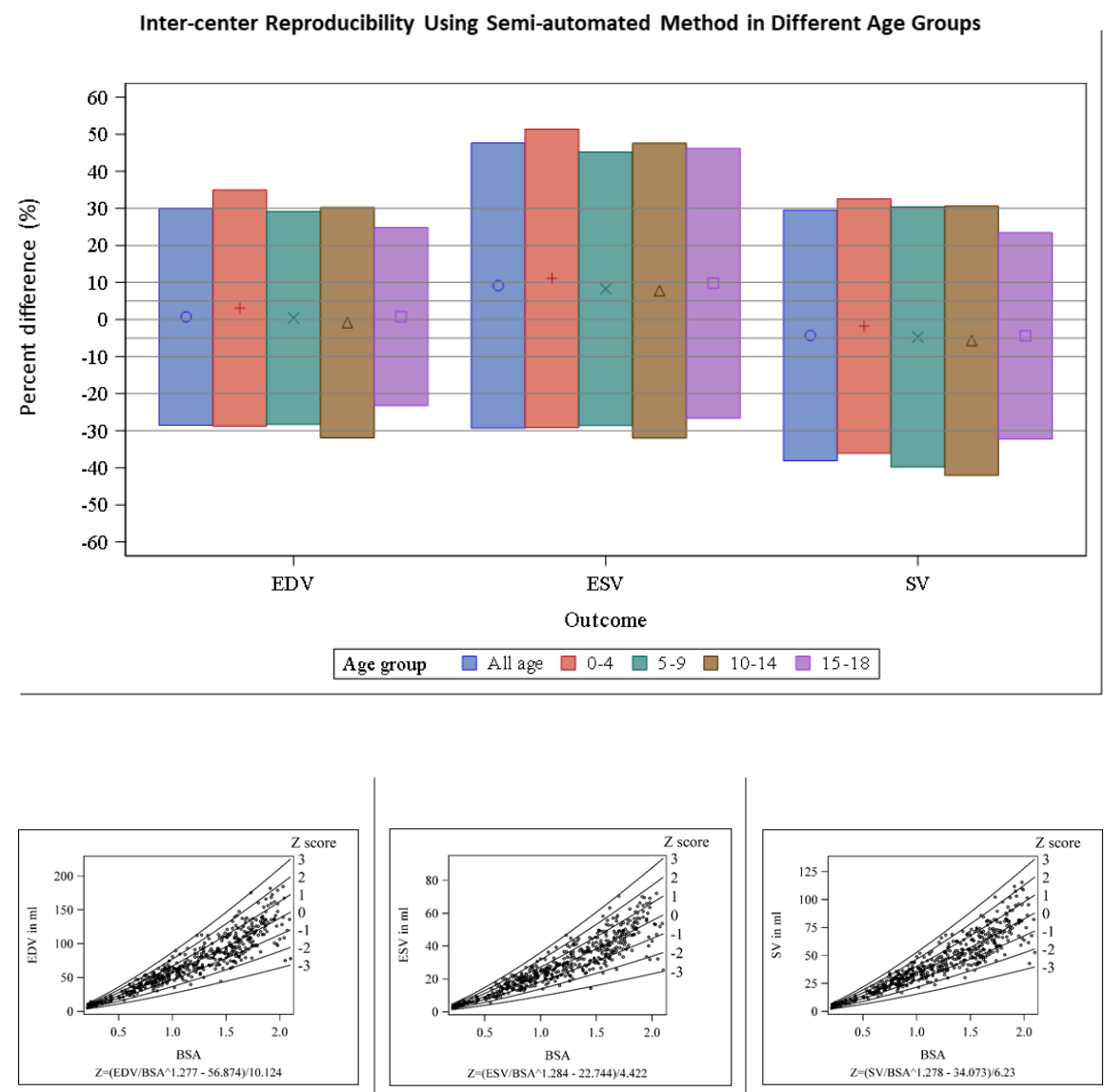